\title{
A lipid pathway for heat adaptation
}

\author{
HUANG Xun \\ State Key Laboratory of Molecular and Developmental Biology, Institute of Genetics and Developmental Biology, Chinese Academy of Sci- \\ ences, Beijing 100101, China
}

Received May 25, 2015; accepted May 28, 2015; published online June 4, 2015

Citation: Huang X. A lipid pathway for heat adaptation. Sci China Life Sci, 2015, 58: 727-728, doi: 10.1007/s11427-015-4880-x

The emergence of cell membrane is an essential step in the origin of life on earth. Cell membrane, a matrix with fatty acid derived lipids and proteins, separates the outside environment from enclosed cell internal. The composition of membrane lipids largely determines biophysical properties of cell membrane, such as fluidity, permeability and deformability, which are essential for cellular processes.

Temperature is an important environmental factor. Different species live in a dramatic variation of temperature conditions; for example, bacterial Thermus Aquaticus (in which the Taq DNA polymerase for PCR was first isolated) grows in hot springs while polar bears reside in arctic area. Individual cell or organism can adapt to a limited range of temperatures, which is also known as thermal tolerance. Temperature changes affect membrane fluidity. It has been known for a long time that bacteria respond to temperature changes by changing the fatty acid saturation of cell membrane through altering the expression of lipid desaturase, a phenomenon named homeoviscous adaptation (HVA) [1]. Later on, the phenomenon of HVA was found conserved in evolution [2]. In bacteria, a two-component regulatory system regulates lipid desaturase expression and mediates HVA. However, the two-component system genes are not found in eukaryotes and the molecular mechanism of HVA remains elusive in eukaryotes.

A recent study by Ma and his colleagues [3] uncovers a novel lipid pathway for HVA in C. elegans. Through a genetic modifier screen for genes affecting lipid desaturase fat-7 reporter expression, eight alleles of acyl-CoA dehy-

email: xhuang@genetics.ac.cn drogenase $a c d h-11$ with elevated fat-7 reporter expression were recovered. Consistent with the role of fat 7 in lipid desaturation, membrane fluidity is increased and the level of stearic acid, the most abundant saturated fatty acid, is reduced in acdh-11 mutants. Importantly, acdh-11 mutants fail to adapt to heat: mutant embryos can develop to adulthood at $15^{\circ} \mathrm{C}$ or $20^{\circ} \mathrm{C}$, but not at $25^{\circ} \mathrm{C}$. Is acdh-11 a regulatory component of HVA? One key feature of the regulatory component is heat responsiveness. Indeed, it was found that heat up-regulates $a c d h-11$ expression. Therefore, the immediate question becomes how acdh-11 regulates lipid desaturase fat-7 expression and heat adaptation. To this end, Ma et al. [3] turned to protein crystal structure analysis and found a strong, specific binding between ACDH-11 and C11/C12 chain fatty acid, but not C8 fatty acid. These findings lead to a hypothesis that "heat-induced ACDH-11 sequesters intracellular $\mathrm{C} 11 / \mathrm{C} 12$-chain fatty acids, which are required for activating nuclear fat-7 expression through fatty acid-regulated transcription factors". Three evidences support this hypothesis. The first one is the effect of various fatty acids on fat-7 expression. $\mathrm{C} 11$ and $\mathrm{C} 12$ fatty acids activate fat-7 expression, while shorter chain fatty acids, C3-C8, or longer chain fatty acids, C13-C20, do not exhibit such effect. Secondly, this effect of $\mathrm{C} 11 / \mathrm{C} 12$ fatty acids is dependent on transcription factor NHR-49. Lastly, the elevated fat-7 expression in acdh-11 mutants is blunted in $n h r-49$ mutants.

The discovery by Ma et al. [3] is significant in that it reveals a novel lipid-related pathway regulating HVA in eukaryotic system. From the evolutionary aspect, compared to HVA in bacteria, this lipid pathway differs from the two-component regulatory system but is similar in the final 
transcriptional control of lipid desaturase. On the other hand, previous reports that the most common inherited disorders of fatty acid oxidation in human caused by $A C D H$ gene mutations, which exhibit exacerbated syndromes under fever or hyperthermia, point to a likely conserved heat adaption mechanism in higher organisms.

Ma et al.'s study depicts a simple model in heat adaptation and more details need to be filled in to have a complete picture. Just list a few here. Is ACDH-11 enzymatic activity required for heat adaptation? Numerous studies have been reported that old enzymes exhibit new functions, which are independent of their enzymatic roles. Along the same line, does $\mathrm{C} 11 / \mathrm{C} 12$ fatty acid or its metabolic products, such as C11/C12-CoA, act as ligand/signal for transcription factor NHR-49? The thermal tolerance of $C$. elegans is different from that of mammals. If the ACDH pathway is conserved in mammals, what determines the thermal tolerance difference? Is the intracellular level of $\mathrm{C} 11 / \mathrm{C} 12$ fatty acid the determinant factor? Without a doubt, more studies are currently under the way.

The mechanism revealed by $\mathrm{Ma}$ et al. raised an interesting point: the level of intracellular free fatty acid, in partic- ular C11/C12 fatty acid, as key determinant for heat adaptation. This lipid pathway is somewhat analog to the role of phosphatidic acid (PA) in $\mathrm{pH}$ sensing in yeast. Normally, PA binds to the transcription factor Opil and prevents its translocation into nucleus. Under low intracellular $\mathrm{pH}$ condition, the change of protonation state of PA unleashes the binding of Opi1, which in turn facilitates the entering of Opi1 into nucleus and represses phospholipid metabolic gene expression [4]. With the coming of a lipid era, there is no doubt that more lipid pathways will be revealed in diverse biological processes.

1 Sinensky M. Homeoviscous Adaptation-A homeostatic process that regulates the viscosity of membrane lipids in Escherichia coli. P Natl Acad Sci USA, 1974, 71: 522-525

2 Cossins AR, Prosser CL. Evolutionary adaptation of membranes to temperature. P Natl Acad Sci USA, 1978, 75: 2040-2043

3 Ma DK, Li Z, Lu AY, Sun F, Chen S, Rothe M, Menzel R, Sun F, Robert HH. Acyl-CoA dehydrogenase drives heat adaptation by sequestering fatty acid. Cell, 2015, 161: 1152-1163

4 Young BP, Shin JJ, Orij R, Chao JT, Li SC, Guan XL, Khong A, Jan E, Wenk MR, Prinz WA, Smits GJ, Loewen CJ. Phosphatidic acid is a $\mathrm{pH}$ biosensor that links membrane biogenesis to metabolism. Science, 2010, 329: 1085-1088

Open Access This article is distributed under the terms of the Creative Commons Attribution License which permits any use, distribution, and reproduction in any medium, provided the original author(s) and source are credited. 